\title{
Extensions of the Longitudinal Envelope Equation
}

\author{
David Neuffer \\ Accelerator Physics Group \\ Fermilab, PO Box 500, Batavia IL 60510
}

\section{Introduction}

Recently, longitudinal space charge effects have become of increased importance in a variety of dynamical situations. The CEBAF FEL injector beam dynamics shows large space-charge effects, even at $10 \mathrm{MeV}(\gamma \approx 20) .{ }^{1} \quad$ Space-charge dominated longitudinal motion has also been studied in the IUCF ion storage ring ${ }^{2,3}$. Previously a longitudinal envelope equation with a selfconsistent phase-space distribution has been developed, ${ }^{4}$ and has been of considerable use in analyzing the motion of these cases. Longitudinal motion in detailed agreement with this envelope equation has been observed at the U. of Maryland Laboratory for Plasma Research, ${ }^{5}$ and at the GSI electron cooling storage ring ESR, ${ }^{6}$ as well as at the IUCF. However, the initial presentation in ref. 4 used non-relativistic linear-accelerator bunching motion as a simplifying approximation in order to avoid inadvertent errors and minimize misprints, and must be adapted to include relativistic and/or synchrotron effects.

In the present note we extend the envelope equation formulae to include relativistic, synchrotron, and acceleration effects, and define the various factors in the equations in explicit detail. The object is to obtain a set of debugged formulae for these extended cases, with all of the various factors defined explicitly, so that the formulae can be used as a reference without repetitive rederivations. The usual ambiguities over emittance definitions and units and $\beta, \gamma, \mathrm{g}$ factors should be resolved. The reader (or readers) is invited to discover any remaining errors, ambiguities or misprints for removal in the next edition.

\section{Nonrelativistic Envelope Equation}

Under simplified assumptions (no transverse dependence, a round beam of radius a within a perfectly conducting round beam chamber of radius $b$, and no resistive wall impedances), the longitudinal space charge force can be written as: ${ }^{7}$

$$
F_{z}=-\frac{q^{2} g}{4 \pi \varepsilon_{0} \gamma^{2}} \frac{d \lambda}{d z}=\frac{d\left(m \gamma \beta_{z} c\right)}{d t}
$$

where $\mathrm{q}$ is the charge of the particles in the bunch (e for electrons, Ze for ions), $\lambda$ is the number of particles per unit length, $\beta, \gamma$ are the usual kinematic factors, and $g=1+2 \ln (\mathrm{b} / \mathrm{a})$ is "a geometrical factor of order unity". Note that $\mathrm{g} \approx 3-4$ in typical accelerators; also, in ref. 3 and some other references, a $g$ factor that is smaller by a factor of 2 is used $\left(g_{3}=1 / 2+\ln (b / a)\right)$. (These expressions for the $\mathrm{g}$ factor ignore radial dependences; observationally Wang et al. observe $\mathrm{g} \approx 2 \ln (\mathrm{b} / \mathrm{a}){ }^{8}{ }^{8}$ ) The force is made non-relativistic simply by taking $\mathrm{E} / \mathrm{m}=\gamma \Rightarrow 1$; that approximation is used in reference 4. (We have used MKS units in eq.1; ref. 4 used cgs units for 
electromagnetic force.)

In addition, there may be a bunching force due to a ramp in a longitudinal accelerating field. This force may be written as:

$$
\mathrm{F}_{\mathrm{z}}=-\mathrm{q} \mathrm{E}_{0}^{\prime}(\mathrm{s}) \mathrm{z}
$$

where $-\mathrm{E}_{0}{ }^{\prime}(\mathrm{s})$ is the local ramp in the longitudinal field and we have chosen to use a linear, position-dependent bunching force. The coordinate $\mathrm{z}$ is position with respect to the bunch center. In reference 4, a self-consistent longitudinal distribution with an envelope equation was developed, consistent with these force equations.

In ref. 4 , the self-consistent longitudinal distribution for a particle bunch is written as:

$$
f\left(z, z^{\prime}, s\right)=\frac{3 N}{2 \pi \varepsilon_{L}} \sqrt{1-\frac{z^{2}}{z_{0}^{2}}-\frac{z_{0}^{2}}{\varepsilon_{L}^{2}}\left(z^{\prime}-\frac{z_{0}^{\prime}}{z_{0}} z\right)^{2}}
$$

within the region in $z, z^{\prime}$ that the argument of the square root is positive ( $f=0$ outside). In reference 4 , position along the accelerator $\mathrm{s}$ is used as the independent variable $\left(\mathrm{z}^{\prime}=\mathrm{dz} / \mathrm{ds}\right) . \mathrm{z}$, $\mathrm{z}^{\prime}$ are particle coordinates, $\mathrm{N}$ is the total number of particles in the bunch. $\mathrm{z}_{0}$ is the bunch halflength (full length $=2 \mathrm{z}_{0}$ ) or beam envelope amplitude. $\varepsilon_{\mathrm{L}}$ is the longitudinal emittance (unnormalized) in $\mathrm{z}-\mathrm{z}^{\prime}$ coordinates. (Lawson ${ }^{8}$ includes an excellent discussion of this distribution but unfortunately his equation 4.49 (in the current edition) contains a typo: the square root is missing. His symbols are also somewhat different: his definition for $\mathrm{N}$ differs and his $\gamma^{\prime \prime}$ refers to the bunching force parameter and not the second derivative of the central beam energy.) The bunch density profile corresponding to eq. 2 is parabolic:

$$
\lambda(\mathrm{z}, \mathrm{s})=\frac{3 \mathrm{~N}}{4 \mathrm{z}_{0}}\left(1-\frac{\mathrm{z}^{2}}{\mathrm{z}_{0}^{2}}\right)
$$

The equation for the beam envelope amplitude $\mathrm{z}_{0}(\mathrm{~s})$ is: 


$$
\frac{\mathrm{d}^{2} \mathrm{z}_{0}}{\mathrm{ds}^{2}}=\frac{\varepsilon_{\mathrm{L}}^{2}}{\mathrm{z}_{0}^{3}}+\frac{3 \mathrm{AN}}{2 \mathrm{z}_{0}^{2}}-\mathrm{K}(\mathrm{s}) \mathrm{z}_{0}
$$

where $A=q^{2} g /\left(4 \pi \varepsilon_{0} m \beta^{2} c^{2}\right)=r_{q} g / \beta^{2} \quad\left(r_{q}\right.$ is the classical particle radius $)$, and $K(s)=q E_{0}{ }^{\prime}(s) /\left(m \beta^{2} c^{2}\right)$ indicates an external linear bunching force. Note that, with this bunch shape and bunching force, the forces on individual particles are linear. We may write:

$$
\frac{\mathrm{d}^{2} \mathrm{z}}{\mathrm{ds}^{2}}=\frac{3 \mathrm{AN}}{2} \frac{\mathrm{z}}{\mathrm{z}_{0}^{3}}-\mathrm{K}(\mathrm{s}) \mathrm{z}
$$

as an equation of motion for individual particles in the bunch. Note that we have dropped relativistic factors in the motion.

\section{Relativistic Envelope Equation}

The above equations are adequate for heavy ions in linear motion. However for protons and electrons, the motion is often somewhat relativistic, and it is desirable to change these equations to include relativistic motion factors. Smith ${ }^{10}$ has given a (non-consistent) relativistic form of the envelope equation; we will rederive it here in a self-consistent form.

It is somewhat tricky to obtain correct relativistic forms in all terms. To improve our chances, we will switch to canonically-correct coordinates from the previous $\mathrm{z}-\mathrm{z}$ ' case, but retain position along the accelerator, $\mathrm{s}$, as our independent variable. Our variables are relative position $\mathrm{z}$ and scaled momentum $\Delta$, where $\Delta=\delta \mathrm{p} /(\mathrm{mc})=\delta(\beta \gamma)$, and $\delta \mathrm{p}$ indicates the difference from the central beam momentum. In these coordinates, the equations of motion are:

$$
\begin{gathered}
\frac{\mathrm{dz}}{\mathrm{ds}}=\frac{\Delta}{\beta \gamma^{3}} \\
\frac{\mathrm{d} \Delta}{\mathrm{ds}}=-\mathrm{K}_{\Delta}(\mathrm{s}) \mathrm{z}-\frac{\mathrm{q}^{2}}{4 \pi \varepsilon_{0} \mathrm{mc}^{2}} \frac{\mathrm{g}}{\beta \gamma^{2}} \frac{\mathrm{d} \lambda(\mathrm{z})}{\mathrm{dz}}
\end{gathered}
$$

where $K_{\Delta}(s)=-q E^{\prime}(s) /\left(\mathrm{m} \mathrm{c}^{2} \beta\right)=\beta \mathrm{K}(\mathrm{s})$.

Following the same method shown in ref. 4 (Take a wild guess and test it.), we can obtain a relativistic envelope equation with a self-consistent distribution from the Vlasov equation. The phase-space distribution function is: 


$$
\mathrm{f}(\mathrm{z}, \Delta, \mathrm{s})=\frac{3 \mathrm{~N}}{2 \pi \varepsilon_{\mathrm{N}}} \sqrt{1-\frac{\mathrm{z}^{2}}{\mathrm{z}_{0}^{2}}-\frac{\mathrm{z}_{0}^{2}}{\varepsilon_{\mathrm{N}}^{2}}\left(\Delta-\frac{\Delta \mathrm{p}_{\mathrm{p}}}{\mathrm{z}_{0}}\right)^{2}}
$$

where $\varepsilon_{\mathrm{N}}$ is the emittance in $\mathrm{z}-\Delta$ coordinates and is invariant (normalized). Here $\mathrm{z}_{0}(\mathrm{~s})$ is the envelope amplitude and $\Delta_{\mathrm{p}}(\mathrm{s})=\beta \gamma^{3}\left(\mathrm{~d} \mathrm{z}_{0} / \mathrm{ds}\right)$. The resulting density profile is parabolic:

$$
\lambda(\mathrm{z}, \mathrm{s})=\frac{3 \mathrm{~N}}{4 \mathrm{z}_{0}}\left(1-\frac{\mathrm{z}^{2}}{\mathrm{z}_{0}^{2}}\right),
$$

which implies a linear space-charge force.

The phase-space distribution is a self-consistent solution of the Vlasov equation:

$$
\frac{\partial \mathrm{f}}{\partial \mathrm{s}}+\mathrm{z}^{\prime} \frac{\partial \mathrm{f}}{\partial \mathrm{z}}+\Delta^{\prime} \frac{\partial \mathrm{f}}{\partial \Delta}=0
$$

if $\mathrm{z}_{0}(\mathrm{~s}), \Delta_{\mathrm{p}}(\mathrm{s})$ are solutions of the envelope equations:

$$
\begin{gathered}
\frac{\mathrm{dz} z_{0}}{\mathrm{ds}}=\frac{\Delta_{\mathrm{p}}}{\beta \gamma^{3}} \\
\frac{\mathrm{d} \Delta_{\mathrm{p}}}{\mathrm{ds}}=\frac{\varepsilon_{\mathrm{N}}^{2}}{\beta \gamma^{3} \mathrm{z}_{0}^{3}}+\frac{3 \mathrm{r}_{\mathrm{q}} \mathrm{gN}}{2 \beta \gamma^{2} \mathrm{z}_{0}^{2}}-\mathrm{K}_{\Delta}(\mathrm{s}) \mathrm{z}_{0}
\end{gathered}
$$

These can be combined into a single envelope equation (found also in $\mathrm{Smith}^{10}$ ):

$$
\frac{\mathrm{d}}{\mathrm{ds}} \beta \gamma^{3} \frac{\mathrm{dz_{0 }}}{\mathrm{ds}}=\frac{\varepsilon_{\mathrm{N}}^{2}}{\beta \gamma^{3} \mathrm{z}_{0}^{3}}+\frac{3 N r_{\mathrm{q}} \mathrm{g}}{2 \beta \gamma^{2} \mathrm{z}_{0}^{2}}-\mathrm{K}_{\Delta}(\mathrm{s}) \mathrm{z}_{0}
$$

Note that the normalized $(\mathrm{z}-\Delta)$ emittance $\varepsilon_{\mathrm{N}}$ is related to the $\left(\mathrm{z}-\mathrm{z}^{\prime}\right)$ emittance $\varepsilon_{\mathrm{L}}$ by $\varepsilon_{\mathrm{N}}=\beta \gamma^{3} \varepsilon_{\mathrm{L}}$. If the central beam energy is unchanged $(\beta, \gamma$ are constant), we can return to unnormalized coordinates, obtaining the envelope equation: 


$$
\frac{\mathrm{d}^{2} \mathrm{z}_{0}}{\mathrm{ds^{2 }}}=\frac{\varepsilon_{\mathrm{L}}^{2}}{\mathrm{z}_{0}^{3}}+\frac{3 \mathrm{NA}}{2 \gamma^{5} \mathrm{z}_{0}^{2}}-\frac{\mathrm{K}(\mathrm{s})}{\gamma^{3}} \mathrm{z}_{0}
$$

which can be compared with the nonrelativistic fixed-energy envelope equation (5). The large factors of $\gamma$ which appear in the force terms indicate that the nonrelativistic approximation becomes somewhat inaccurate at relatively low energies for light particles. It is therefore more desirable to use the relativistic forms. (The appearance of larger powers of $\gamma$ in longitudinal motion can be explained in terms of the appearance of a longitudinal mass $\left(\mathrm{m} \gamma^{3}\right)$ rather than a transverse mass $(\mathrm{m} \gamma)$ in the equations of motion. ${ }^{8}$ From eq. 7 , we note that $\mathrm{dz} / \mathrm{dt}=\delta \mathrm{p} /\left(\mathrm{m} \gamma^{3}\right)$.)

An important parameter is the momentum width (or energy width) of the beam. $\mathrm{z}_{0}$ is the bunch half-width, but $\Delta_{\mathrm{p}}$ refers to $\beta \gamma^{3} \mathrm{dz}_{0} / \mathrm{ds}$, in our current notation. A complementary momentum halfwidth $\Delta_{0}$ can be obtained. A derivation for this expression can be obtained by rewriting the argument of the distribution function as:

$$
1-\frac{\mathrm{z}^{2}}{\mathrm{z}_{0}^{2}}-\frac{\mathrm{z}_{0}^{2}}{\varepsilon_{\mathrm{N}}^{2}}\left(\Delta-\frac{\Delta_{\mathrm{p}}}{\mathrm{z}_{0}} \mathrm{z}\right)^{2}=1-\frac{\varepsilon_{\mathrm{N}}^{2}+\Delta_{\mathrm{p}}^{2} \mathrm{z}_{0}^{2}}{\varepsilon_{\mathrm{N}}^{2} \mathrm{z}_{0}^{2}}\left(\mathrm{z}-\frac{\Delta_{\mathrm{p}} \mathrm{z}_{0}^{3}}{\varepsilon_{\mathrm{N}}^{2}+\Delta_{\mathrm{p}}^{2} \mathrm{z}_{0}^{2}} \Delta\right)^{2}-\frac{\mathrm{z}_{0}^{2}}{\varepsilon_{\mathrm{N}}^{2}+\Delta_{\mathrm{p}}^{2} \mathrm{z}_{0}^{2}} \Delta^{2}
$$

The maximum value of $\Delta, \Delta_{0}$, in this distribution is found from:

$$
\Delta_{0}^{2}=\frac{\varepsilon_{\mathrm{N}}^{2}}{\mathrm{z}_{0}^{2}}+\Delta_{\mathrm{p}}^{2}=\frac{\varepsilon_{\mathrm{N}}^{2}}{\mathrm{z}_{0}^{2}}+\beta^{2} \gamma^{6}\left(\frac{\mathrm{dz}}{\mathrm{ds}}\right)^{2}
$$

This can be written in terms of the relative momentum spread (half-width $\delta p / p$ ) as:

$$
\left(\frac{\delta \mathrm{p}}{\mathrm{p}}\right)^{2}=\frac{\varepsilon_{\mathrm{N}}^{2}}{\beta^{2} \gamma^{2} \mathrm{z}_{0}^{2}}+\gamma^{4}\left(\frac{\mathrm{dz}}{\mathrm{ds}}\right)^{2}
$$

The large factors of $\gamma$ in the expression indicate that in semi-relativistic motion a relatively large momentum spread increase can occur with relatively small envelope-size motion.

\section{Circular Accelerator (Synchrotron) Motion}

The equations of motion are somewhat modified in the case of motion in a circular accelerator. In circular motion, the change in particle position with respect to time also depends on the change in path length with momentum, and those changes in path length result from the 
momentum-dependence of particle motion through the bending magnets and depend on the accelerator transport design. That dependence is expressed in terms of the ring momentum compaction factor $\alpha_{\mathrm{p}}=1 / \gamma_{\mathrm{T}}^{2}$, where $\gamma_{\mathrm{T}}$ is the "transition gamma". The equation of motion is changed by:

$$
\frac{\mathrm{dz}}{\mathrm{ds}}=\left(\frac{1}{\gamma^{2}}-\frac{1}{\gamma_{\mathrm{T}}^{2}}\right) \frac{\delta \mathrm{p}}{\mathrm{p}}=\left(\frac{1}{\gamma^{2}}-\frac{1}{\gamma_{\mathrm{T}}^{2}}\right) \frac{\Delta}{\beta \gamma}=\eta_{\mathrm{p}} \frac{\Delta}{\beta \gamma}
$$

where we have introduced the symbol $\eta_{\mathrm{p}}=1 / \gamma^{2}-1 / \gamma_{\mathrm{T}}^{2}$.

Synchrotrons have an accelerating rf voltage per turn, $\mathrm{V}_{0} \sin (\phi)=\mathrm{V}_{0} \sin (\mathrm{h} \mathrm{z} / \mathrm{R})$, which provides acceleration and bunching, where $\phi$ is the $\mathrm{rf}$ phase, $\mathrm{h}$ is the $\mathrm{rf}$ harmonic number and $2 \pi \mathrm{R}$ is the ring circumference. In a linearized (short-bunch) approximation, this can be decomposed into the acceleration of the bunch center $\left(\mathrm{d} \gamma / \mathrm{ds}=\mathrm{e} \mathrm{V}_{0} \sin \left(\phi_{\mathrm{s}}\right) /\left(2 \pi \mathrm{Rmc}^{2}\right)\right)$ and a bunching term. The bunching term can be written as:

$$
\frac{\mathrm{d} \Delta}{\mathrm{ds}}=\frac{\mathrm{eV}_{0} \cos \left(\phi_{\mathrm{s}}\right) \mathrm{h}}{2 \pi \mathrm{R}^{2} \beta m \mathrm{c}^{2}} \mathrm{z}=-\mathrm{K}_{\mathrm{s}} \mathrm{z}
$$

Developing an envelope equation in exactly the same manner as above obtains the equations:

$$
\begin{gathered}
\frac{d z_{0}}{d s}=\eta_{p} \frac{\Delta_{s}}{\beta \gamma} \\
\frac{d \Delta_{s}}{d s}=\eta_{p} \frac{\varepsilon_{N}^{2}}{\beta \gamma z_{0}^{3}}+\frac{3 r_{q} g N}{2 \beta \gamma^{2} z_{0}^{2}}-K_{s} z_{0}
\end{gathered}
$$

where we have used the symbol $\Delta_{\mathrm{s}}=\beta \gamma / \eta_{\mathrm{p}}\left(\mathrm{dz} \mathrm{z}_{0} / \mathrm{ds}\right)$ instead of $\Delta_{\mathrm{p}}$ to distinguish synchrotron from linear motion. The distribution function would be the same as before, but with $\Delta_{\mathrm{p}}$ replaced by $\Delta_{\mathrm{S}}$

If the acceleration term is zero, this can be simplified, as above, to:

$$
\frac{\mathrm{d}^{2} \mathrm{z}_{0}}{\mathrm{ds}^{2}}=\eta_{\mathrm{p}}^{2} \frac{\varepsilon_{\mathrm{N}}^{2}}{\beta^{2} \gamma^{2} \mathrm{z}_{0}^{3}}+\frac{3 \eta_{\mathrm{p}} \mathrm{r}_{\mathrm{q}} \mathrm{gN}}{2 \beta^{2} \gamma^{3} \mathrm{z}_{0}^{2}}-\frac{\eta_{\mathrm{p}} \mathrm{K}_{\mathrm{s}}}{\beta \gamma} \mathrm{z}_{0}
$$

Nagaitsev et $\mathrm{al}^{3}$ have obtained an envelope equation for synchrotron motion but in somewhat different coordinates(t, scaled position, and relative momentum $\delta=\delta \mathrm{p} / \mathrm{p}$ ) We will transform these equations toward that form by using $\mathrm{dt}=\mathrm{ds} / \beta \mathrm{c}$ and scaled position $\mathrm{Z}=\mathrm{z}_{0} / \beta \mathrm{R}$ as variables, 
obtaining:

$$
\frac{d^{2} Z}{d t^{2}}=\left(\frac{c}{R}\right)^{2}\left(\frac{\eta_{p}^{2} \varepsilon_{N}^{2}}{R^{2} \beta^{4} \gamma^{2} Z^{3}}+\frac{3 \eta_{p} r_{q} g N}{2 R \beta^{3} \gamma^{3} Z^{2}}-\frac{\eta_{p} h e V_{B}}{2 \pi m c^{2} \gamma} Z\right)
$$

We have redefined the rf voltage, using $\mathrm{V}_{\mathrm{B}}=-\mathrm{V}_{0} \sin \left(\phi_{\mathrm{s}}\right)$, to obtain the desired sign in that term. This equation can be simplified in form by changing emittance to Z- $\delta$ units $\left(\varepsilon_{\mathrm{Z}-\delta}=\varepsilon_{\mathrm{N}} /\left(\mathrm{R} \beta^{2} \gamma\right)\right)$, expressing space charge in terms of the bunch current $I=e \beta c N /(2 \pi R), I_{p}=e c \beta^{4} \gamma^{3} /\left(3 \pi g \eta_{p} r_{q}\right), A$ $=\eta_{\mathrm{p}} \mathrm{heV}_{\mathrm{B}} /\left(2 \pi \mathrm{mc}^{2} \gamma\right)$ :

$$
\frac{\mathrm{d}^{2} \mathrm{Z}}{\mathrm{dt}^{2}}=\left(\frac{\mathrm{c}}{\mathrm{R}}\right)^{2}\left(\frac{\eta_{\mathrm{p}}^{2} \varepsilon_{\mathrm{Z} \delta}^{2}}{\mathrm{Z}^{3}}+\frac{\mathrm{I}}{\mathrm{I}_{\mathrm{p}} \mathrm{Z}^{2}}-\mathrm{A} \mathrm{Z}\right)
$$

This is similar to the form used in ref 3 , eq. 11, but that form uses rms full-widths for bunch length and emittance, hence differ by factors of $\sqrt{ } 2$ and 2 in the formulae. $g$ is also defined differently by a factor of two, and $\eta_{p}$ is handled differently. After these appropriate unit changes, the expressions agree. Note that the sign of $\eta_{p}$ is not handled in ref. 3 but is handled correctly here (I think); space charge actually bunches the beam when the beam energy is above transition $\left(\gamma>\gamma_{\mathrm{T}}\right)$

\section{Discussion}

The envelope equations described here can be used as an accurate first approximation for longitudinal motion in any accelerator. As mentioned above, experimental groups at Maryland, IUCF, and GSI have all observed longitudinal motion and envelope oscillations in very close agreement with the present model. The self-consistent longitudinal distribution has been used to initiate a modal analysis as a basis for instability studies ${ }^{11}$ ( as has also been done for transverse motion, based on the Kapchinsky - Vladimirsky equation ${ }^{12}$ ). The dipole, quadrupole, and sextupole modes have been observed at GSI, in close agreement with the predictions. ${ }^{6}$

\section{References}

1. P. Liger, G. A. Krafft and D. Neuffer, Nucl. Inst. and Meth. A318, p. 290 (1992).

2. T. P. Ellison, S S Nagaitsev, M. S. Ball, D. D. Caussyn, M. J. Ellison, and B. J. Hamilton, Phys. Rev. Lett. 70, p. 790 (1993).

3. S. Nagaitsev, T. Ellison, M. Ellison, D. Anderson, "The Investigation of Space Charge Dominated Cooled Bunched Beams in a Synchrotron", Proc. Beam Cooling Workshop, Montreux, 1993.

4. D. Neuffer, IEEE Trans. Nucl. Sci. NS-26, 3031 (1979). 
5. D. X. Wang, J. G. Wang, D. Kehne, and M. Reiser, Appl. Phys. Lett. 62, p. 25 (1993).

6. I. Hofmann, presented at the 1994 European Particle Accelerator Conference (1994).

7. A. Hofmann, in CERN 77-13, p. 139 (CERN, Geneva, 1977).

8. J. D. Lawson, The Physics of Charged Particle Beams, Oxford University Press, New York, 1988).

9. J. G. Wang, H. Suk, D. X. Wang, and M. Reiser, submitted to Phys. Rev. Lett. (1994).

10. L. Smith, in ERDA Summer study for Heavy Ion Inertial Fusion, LBL 5543, p. 77 (1976).

11. D. Neuffer, Particle Accelerators 11, p. 23 (1980).

12. I. Hofmann, L. J. Laslett, L. Smith, I. Haber, Particle Accelerators 13, p.145 (1983). 